\title{
A Photometric study of the near-contact system RU Ursae Minoris
}

\author{
V. N. Manimanis and P. G. Niarchos \\ Department of Astrophysics, Astronomy and Mechanics, Faculty of Physics, University of Athens, \\ 15784 Zographou, Athens, Greece \\ Received 7 December 2000 / Accepted 23 January 2001

\begin{abstract}
The near-contact system RU UMi with an F0 primary and a K5 secondary was observed in $U, B$ and $V$ wavelengths in May and July 1998, as well as in March, May and July 1999. Six new observed times of minima are given and a new ephemeris is proposed. The basic parameters of the system extracted by our observations were used for spot modelling of the light curves. A simple spot distribution was determined, based on a model with one relatively small cool spot on the surface of the secondary. Absolute elements were calculated and the evolutionary status was determined. Our data favor a semi-detached configuration, with the secondary filling its inner Roche lobe; the primary must also be near the limits of its lobe.
\end{abstract}

Key words. stars: binaries: eclipsing, starspots, stars: individual: RU UMi

\section{Introduction}

RU Ursae Minoris (BD $\left.+70^{\circ} 0751\right)$ was discovered as an eclipsing variable star by Strohmeier \& Knigge (1960). After its discovery, numerous observations of the system have been made by various observers. Summarized observational aspects and light curves of RU UMi have been reported by Wood (1971), Nha (1973), De Bernardi (1977), Okazaki et al. (1988), Rafert (1990), Bell et al. (1993) and more recently by Maxted \& Hilditch (1996). A photometric solution has been also determined by Kaluzny (1985). A spectroscopic orbit of the system was obtained by Maxted \& Hilditch (1996). RU UMi is an eclipsing binary that can be classified as a near-contact or semidetached system. Shaw includes it in both his first (1990) and his second (1994) catalog of near-contact systems as one whose subclass, FO Vir or V1010 Oph, is unknown. According to Shaw (1990), as FO Vir type systems are defined those that have a primary component of normal size inside its Roche lobe and a 2 to 3 times oversized secondary at or near its Roche lobe. V1010 Oph are defined as those with a normal primary at or near its Roche lobe and an up to 1.5 times oversized secondary well inside its Roche lobe. Members of the latter subclass often show asymmetrical light curves, while the members of the FO Vir subclass never do. Therefore, we observed and an-

Send offprint requests to: P. G. Niarchos,

e-mail: pniarcho@cc.uoa.gr

* Table 2 is only available in electronic form at the CDS via anonymous ftp to cdsarc.u-strabg.fr(130.79.128.5) or via http://cdsweb.u-strasbg.fr/cgi-bin/qeat?/A+A/369/960 alyzed the system in order to determine, among others, its subclass and evolutionary state.

\section{Observations}

The photoelectric photometry observations of RU UMi were made over 12 spring and summer nights in 1998 and 1999, namely on May 26-28 and July 19-24 of 1998 and also on March 13-14, May 13-14 and July 8-11 of 1999. (Some low-quality data taken during the night of March 12-13, 1999, were omitted from the subsequent analysis.) The instruments used were the $122-\mathrm{cm}$ Cassegrain reflector at the Kryonerion Station of the National Observatory of Athens and a pulse-counting photoelectric photometer. The three intermediate pass-band filters used were selected to be in close accordance with the standard color system $U B V$, however the data have not been transformed to the standard system. The stars $\mathrm{BD}+70^{\circ} 749$ and $\mathrm{BD}+70^{\circ} 754$ were used as comparison and check stars, respectively. A total number of 699 observations were obtained in each color, 329 from the 7 nights of 1998 and 370 from the 5 nights of 1999 . The photometry software coupled with the photometer calculates the nightly extinction coefficients for each filter and automatically corrects for differential extinction. The probable error of a single observation was estimated to be $\pm 0.010 \mathrm{mag}$ in yellow and $\pm 0.012 \mathrm{mag}$ in blue and ultraviolet. Figure 1 shows all the observations made in the two-year period. Individual observations are available in electronic form upon request. 

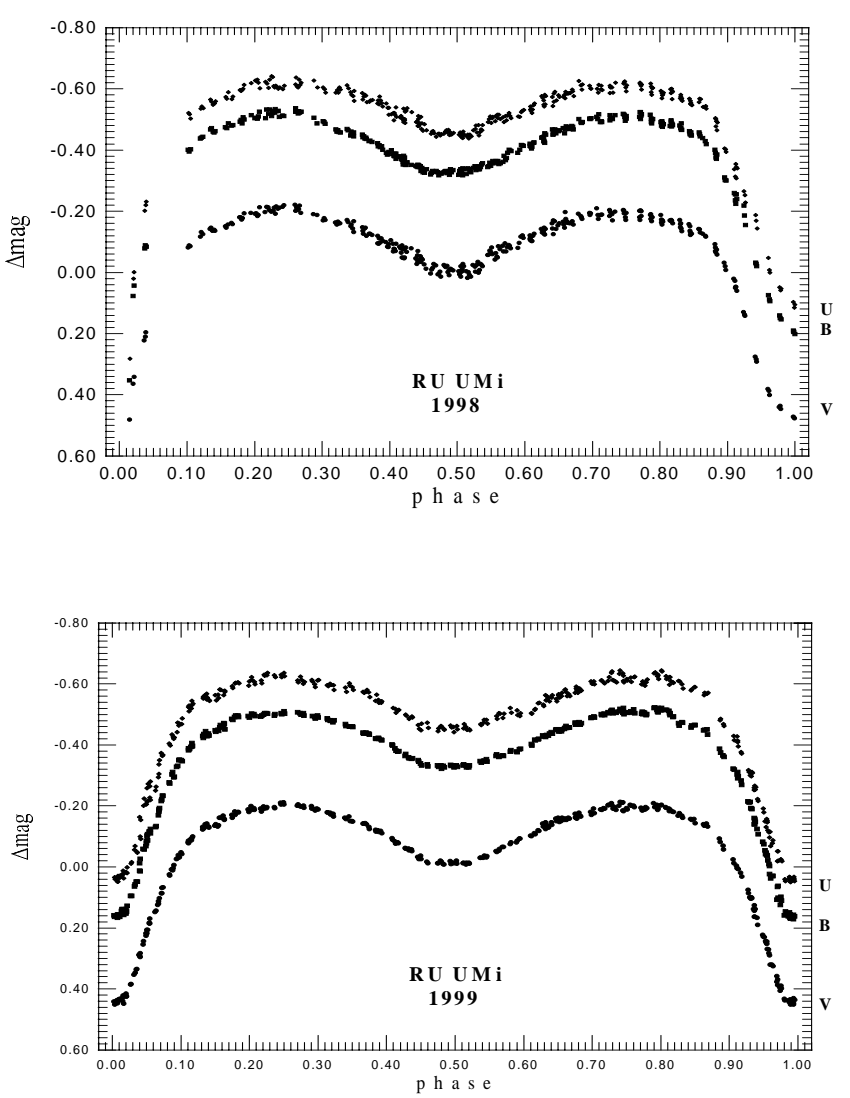

Fig. 1. The observed light curves of RU UMi in 1998 (upper) and 1999 (lower)

Table 1. New times of minima of RU UMi

\begin{tabular}{lcccc}
\hline $\begin{array}{l}\text { HJD } \\
(2400000+)\end{array}$ & Error & $E$ & Filter & $\begin{array}{c}\text { Type } \\
\text { of minimum }\end{array}$ \\
\hline 50960.4870 & 0.0012 & -554.0 & $V, B, U$ & I \\
51015.3351 & 0.0025 & -449.5 & $V, B, U$ & II \\
51251.2970 & 0.0005 & 0.0 & $V, B, U$ & I \\
51251.5587 & 0.0006 & 0.5 & $V, B, U$ & II \\
51368.3539 & 0.0014 & 223.0 & $V, B, U$ & I \\
51369.4031 & 0.0014 & 225.0 & $V, B, U$ & I \\
\hline
\end{tabular}

\section{Times of minima}

Six new times of primary and secondary minima were obtained from our observations, and they were calculated using the method of Kwee \& van Woerden (1956). Three different times for each minimum were computed, one for each color, and these were combined to give the values listed in Table 1.

From our minima times the following new ephemeris has been derived by a linear least square fit:

$$
\begin{array}{r}
\text { J.D.hel. (Min I })=2451251.2951+0.5249295 \cdot E \\
\pm 0.0012 \pm 0.0000039 .
\end{array}
$$

No indication of period change can be inferred by comparing our ephemeris with the more recent ephemeris by Bell et al. (1993).

\section{Unspotted solution}

RU UMi exhibits a light curve with the first and the second quadrature equally bright. Our light curves do not show an O' Connell effect, as one can see better in the higher-quality 1999 curves. If such an effect is present, the difference between the two maxima is smaller than the error in all light curves. As a first approximation, we solved the light curves assuming that there are no spots on the components of the system. 100 normal points for each color and year were formed from the individual observations and assigned weight equal to the number of observations per point. These points for the 1999 observations, together with their respective weights, are given in Table 2. From an inspection of the curves, we decided that the observations in the phase interval 0.55 to 0.92 should be excluded, as this part of the light curve could be slightly affected by the presence of spots (as suggested by Maxted \& Hilditch 1996). The subscripts 1 and 2 refer to the star being eclipsed at primary and secondary minimum, respectively. The 1996 version of the WilsonDevinney code was used in the light curve analysis.

A starting set of input parameters for the unspotted solution was adopted from the values of Rafert (1990). A $q$-search gave a starting value for $q=0.4$; the adjustable parameters for the main runs were: the conjunction phase $\phi$, the inclination $i$, the temperature $T_{2}$ of the secondary, the non-dimensional surface potential $\Omega$, the mass-ratio $q=M_{2} / M_{1}$ and the non-normalized monochromatic luminosities $L_{1}\left(L_{1 V}, L_{1 B}\right.$ and $\left.L_{1 U}\right)$. The fixed parameter was the temperature $T_{1}$, adopted from the value given by Rafert (1990). Assumed values for limb darkening (Van Hamme 1993), gravity darkening and bolometric albedos, according to the spectral types of the components, were used, while the third light was assumed to be zero. The results converged only when Mode 5 was used, i.e. the mode for the case when the secondary star exactly fills its limiting lobe. In this case, the surface potential $\Omega_{1}$ is a free (adjustable) parameter, but not $\Omega_{2}$. The reverse is true for Mode 4 . The results are given in Table 3. Besides Mode 4, Modes 2 and 3 were also tried, without achieving convergence.

Our final values of $q$ are in good agreement with the spectroscopic mass ratio obtained by Maxted and Hilditch. However, they adopt a lower $T_{1}$ value of $7200 \mathrm{~K}$.

\section{Spot modelling}

The parameters found in the unspotted solution for the 1999 light curve were subsequently used in the Binary Maker 2.0 program (Bradstreet 1993) to model the light curves with spots. This program allows up to six spots to be placed on the surface of the components. However, since there were no major observed variations and asymmetries of the light curves, we adopted the simplest possible model, placing only one cool spot on the surface of the cool secondary, since the temperature of the primary is, in theory, too high to allow spots. The spot modelling 
Table 3. Unspotted light curve solutions of RU UMi $(B, V$ and $U$ curves)

\begin{tabular}{|c|c|c|}
\hline Parameter & 1998 & 1999 \\
\hline$\phi_{0}$ & $0.0037 \pm 0.0002$ & $0.9982 \pm 0.0001$ \\
\hline$i$ (degrees) & $84.18 \pm 0.37$ & $82.59 \pm 0.12$ \\
\hline$g_{1}$ & $1.00^{*}$ & $1.00^{*}$ \\
\hline$g_{2}$ & $0.32^{*}$ & $0.32^{*}$ \\
\hline$T_{1}(\mathrm{~K})$ & $7500^{*}$ & $7500^{*}$ \\
\hline$T_{2}(\mathrm{~K})$ & $4790 \pm 24$ & $4658 \pm 18$ \\
\hline$A_{1}$ & $1.0^{*}$ & $1.0^{*}$ \\
\hline$A_{2}$ & $0.5^{*}$ & $0.5^{*}$ \\
\hline$\Omega_{1}$ & $2.6485 \pm 0.0051$ & $2.6336 \pm 0.0020$ \\
\hline$\Omega_{2}$ & 2.4758 & 2.5079 \\
\hline$q=M_{2} / M_{1}$ & $0.3054 \pm 0.0029$ & $0.3190 \pm 0.0012$ \\
\hline$L_{1} /\left(L_{1}+L_{2}\right)(U)$ & $0.9773 \pm 0.0035$ & $0.9817 \pm 0.0023$ \\
\hline$L_{1} /\left(L_{1}+L_{2}\right)(B)$ & $0.9671 \pm 0.0024$ & $0.9726 \pm 0.0015$ \\
\hline$L_{1} /\left(L_{1}+L_{2}\right)(V)$ & $0.9479 \pm 0.0021$ & $0.9548 \pm 0.0015$ \\
\hline$X_{1}($ bolo $)$ & $0.54^{*}$ & $0.54^{*}$ \\
\hline$X_{2}$ (bolo) & $0.47^{*}$ & $0.47^{*}$ \\
\hline$X_{1}(U)$ & $0.79^{*}$ & $0.79^{*}$ \\
\hline$X_{2}(U)$ & $0.97^{*}$ & $0.97^{*}$ \\
\hline$X_{1}(B)$ & $0.69^{*}$ & $0.69^{*}$ \\
\hline$X_{2}(B)$ & $0.87^{*}$ & $0.87^{*}$ \\
\hline$X_{1}(V)$ & $0.51^{*}$ & $0.51^{*}$ \\
\hline$X_{2}(V)$ & $0.71^{*}$ & $0.71^{*}$ \\
\hline$r_{1}$ (volume) & $0.4445 \pm 0.0010$ & $0.4511 \pm 0.0005$ \\
\hline$r_{1}($ pole $)$ & $0.4226 \pm 0.0008$ & $0.4273 \pm 0.0004$ \\
\hline$r_{1}$ (point) & $0.4895 \pm 0.0020$ & $0.5042 \pm 0.0012$ \\
\hline$r_{1}$ (side) & $0.4467 \pm 0.0010$ & $0.4530 \pm 0.0005$ \\
\hline$r_{1}$ (back) & $0.4635 \pm 0.0013$ & $0.4718 \pm 0.0006$ \\
\hline$r_{2}$ (volume) & $0.2824 \pm 0.0007$ & $0.2849 \pm 0.0003$ \\
\hline$r_{2}($ pole $)$ & $0.2622 \pm 0.0007$ & $0.2656 \pm 0.0003$ \\
\hline$r_{2}$ (point) & $0.3804 \pm 0.0016$ & $0.3850 \pm 0.0013$ \\
\hline$r_{2}$ (side) & $0.2730 \pm 0.0007$ & $0.2767 \pm 0.0003$ \\
\hline$r_{2}$ (back) & $0.3057 \pm 0.0007$ & $0.3094 \pm 0.0003$ \\
\hline$\Sigma(\text { res })^{2}$ & 0.0687 & 0.0368 \\
\hline
\end{tabular}

was derived based on the $U, B$, and $V$ light curves, by fitting the theoretical to the observational light curves using Binary Maker 2.0 and subsequently refining the spot parameters with the Wilson-Devinney code. The parameters of the sole spot are: latitude $0^{\circ}$ (assumed), longitude $172.31^{\circ} \pm 9.65^{\circ}$, spot radius $13.41^{\circ} \pm 0.97^{\circ}$ and temperature factor $0.803 \pm 0.052$. The other parameters of the spotted model are given in Table 4 . The longitude of the spot near $180^{\circ}$ agrees with the suggestion by Maxted and Hilditch that large-scale spot activity is concentrated on the averted hemisphere of the secondary component of the system.

However, we feel that this fact can equally well suggest that no spots are present, but rather that the inclination of the system is higher, as in the unspotted solution; note that $\Sigma(\text { res })^{2}$ in the case of the spotted solution is practically the same as in the case of the unspotted one for 1999. Moreover, the existence of spots on the surface of the secondary, which contributes a few percent of the total light of the system, cannot be expected to have a measurable influence on the shape of the light curve. Theoretical light curves based on the unspotted and spotted solution
Table 4. Spotted light curve solution of RU UMi ( $B, V$ and $U$ curves)

\begin{tabular}{|c|c|}
\hline Parameter & 1999 \\
\hline$\phi_{0}$ & $0.9985 \pm 0.0001$ \\
\hline$i$ (degrees) & $82.48 \pm 0.12$ \\
\hline$g_{1}$ & $1.00^{*}$ \\
\hline$g_{2}$ & $0.32^{*}$ \\
\hline$T_{1}(\mathrm{~K})$ & $7500^{*}$ \\
\hline$T_{2}(\mathrm{~K})$ & $4666 \pm 20$ \\
\hline$A_{1}$ & $1.0^{*}$ \\
\hline$A_{2}$ & $0.5^{*}$ \\
\hline$\Omega_{1}$ & $2.6296 \pm 0.0018$ \\
\hline$\Omega_{2}$ & 2.5072 \\
\hline$q=M_{2} / M_{1}$ & $0.3187 \pm 0.0013$ \\
\hline$L_{1} /\left(L_{1}+L_{2}\right)(U)$ & $0.9816 \pm 0.0024$ \\
\hline$L_{1} /\left(L_{1}+L_{2}\right)(B)$ & $0.9725 \pm 0.0016$ \\
\hline$L_{1} /\left(L_{1}+L_{2}\right)(V)$ & $0.9548 \pm 0.0015$ \\
\hline$X_{1}$ (bolo) & $0.54^{*}$ \\
\hline$X_{2}$ (bolo) & $0.47^{*}$ \\
\hline$X_{1}(U)$ & $0.79^{*}$ \\
\hline$X_{2}(U)$ & $0.97^{*}$ \\
\hline$X_{1}(B)$ & $0.69^{*}$ \\
\hline$X_{2}(B)$ & $0.87^{*}$ \\
\hline$X_{1}(V)$ & $0.51^{*}$ \\
\hline$X_{2}(V)$ & $0.71^{*}$ \\
\hline$r_{1}$ (volume) & $0.4519 \pm 0.0005$ \\
\hline$r_{1}($ pole $)$ & $0.4280 \pm 0.0004$ \\
\hline$r_{1}$ (point) & $0.5057 \pm 0.0012$ \\
\hline$r_{1}$ (side) & $0.4538 \pm 0.0005$ \\
\hline$r_{1}$ (back) & $0.4728 \pm 0.0007$ \\
\hline$r_{2}$ (volume) & $0.2850 \pm 0.0003$ \\
\hline$r_{2}($ pole $)$ & $0.2656 \pm 0.0003$ \\
\hline$r_{2}$ (point) & $0.3849 \pm 0.0014$ \\
\hline$r_{2}$ (side) & $0.2766 \pm 0.0003$ \\
\hline$r_{2}($ back $)$ & $0.3093 \pm 0.0003$ \\
\hline$\Sigma(\text { res })^{2}$ & 0.0371 \\
\hline
\end{tabular}

along with the observed ones (normal points) are shown in Fig. 2. Three-dimensional models of the system with the spot visible at phase 0 are given in Fig. 3, while in Fig. 4 we give an outline of the configuration of the system.

\section{State of evolution}

From our solution and from the spectroscopic orbit by Maxted and Hilditch for a radiative atmosphere of the primary, we obtained the following absolute elements in solar units:

$$
\begin{array}{ll}
R_{1}=1.87 \pm 0.01 & R_{2}=1.18 \pm 0.01 \\
L_{1}=9.87 \pm 0.51 & L_{2}=0.59 \pm 0.01 \\
M_{1}=2.66 \pm 0.13 & M_{2}=0.79 \pm 0.05
\end{array}
$$

and the bolometric absolute magnitudes:

$M_{\mathrm{bol}(1)}=2.26 \quad$ and $\quad M_{\mathrm{bol}(2)}=5.33$.

According to them, the primary component is located relatively close to the ZAMS line (for stars of solar metallicity) in the mass-radius diagram, indicating an almost 

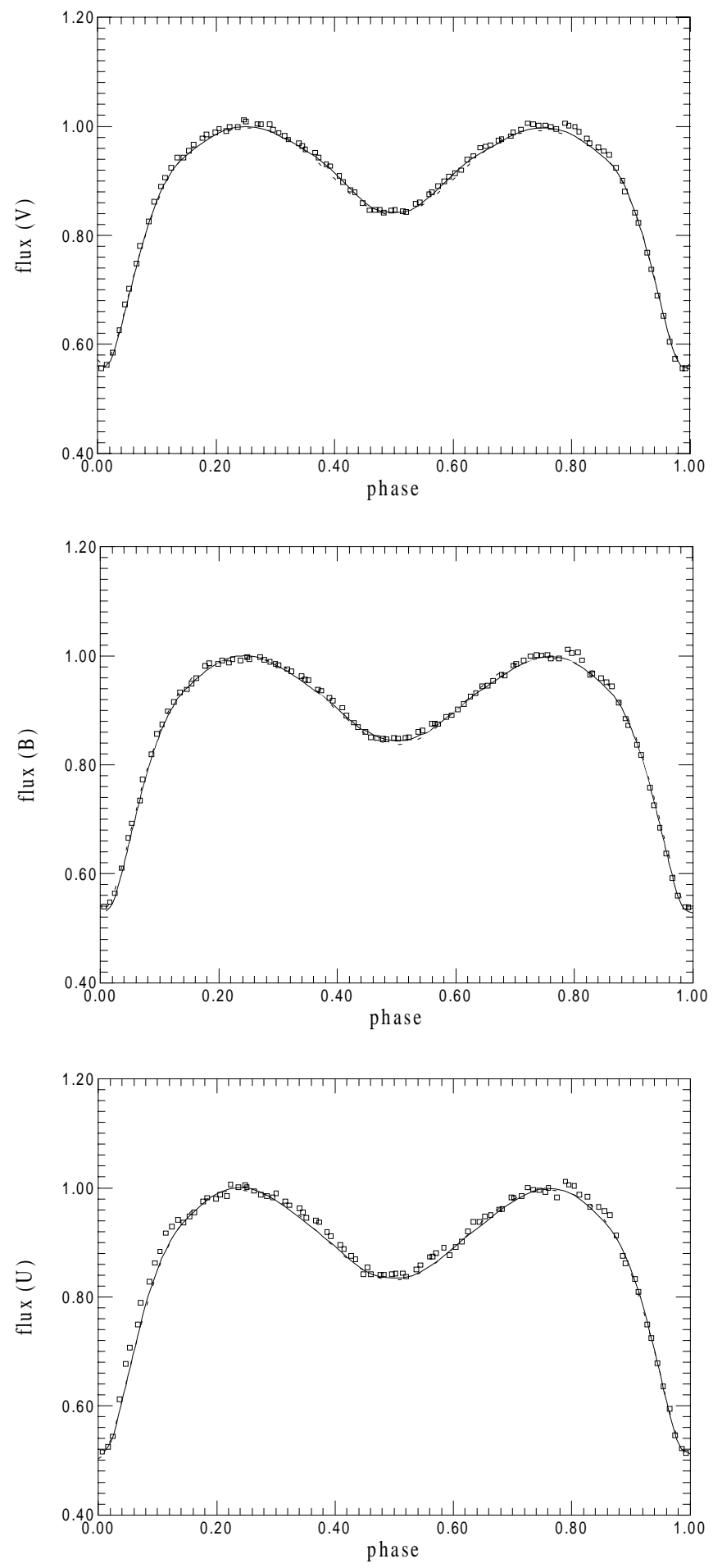

Fig. 2. Normal points and theoretical light curves of RU UMi for 1999. The dashed line (barely seen) corresponds to the unspotted solution, while the solid line corresponds to the spotted solution

unevolved star, while the secondary component seems to have evolved beyond the TAMS line, occupying an intermediate position among the secondaries of the sample of Hilditch et al. (1988). More extreme is the position of the primary in the mass-luminosity diagram, indicating a totally unevolved star (always assuming solar metallicities),

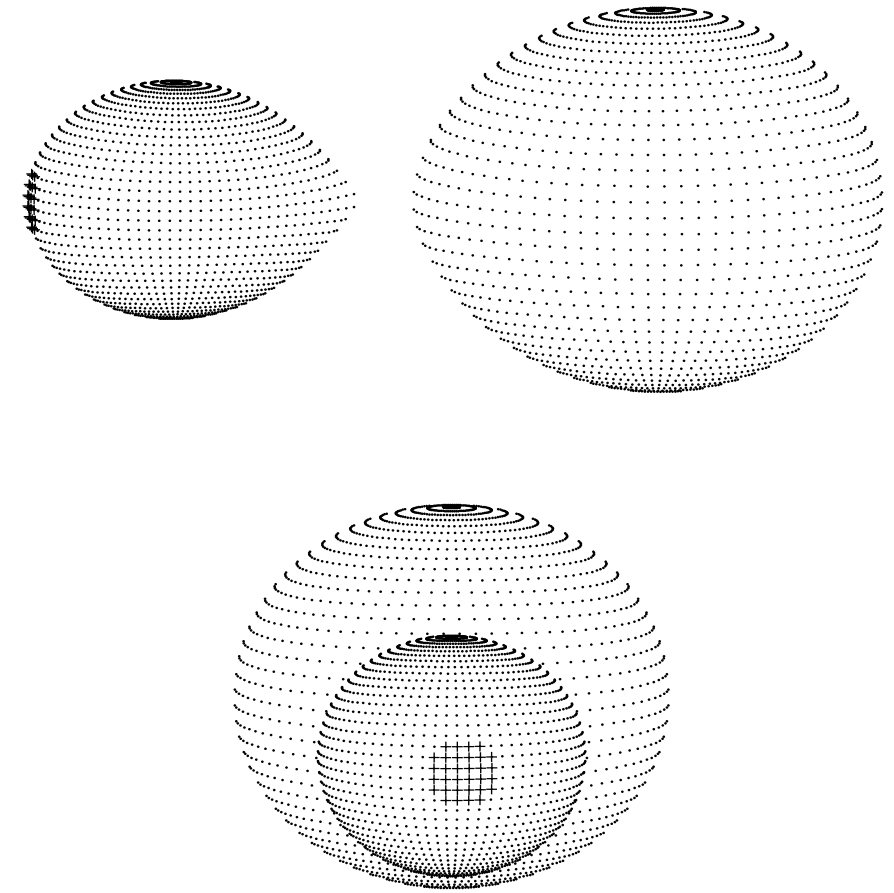

Fig. 3. Three-dimensional models of RU UMi for 1999, corresponding to phases 0.75 (upper) and 0.0 (lower)

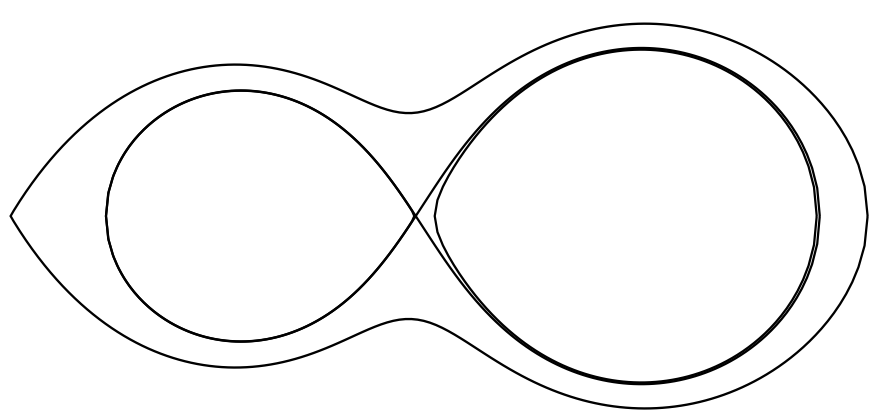

Fig. 4. Surface outlines of the RU UMi binary system for our 1999 solution

the secondary appearing also somewhat less evolved than in the $\mathrm{M}-\mathrm{R}$ diagram. On the other hand, in the $\mathrm{H}-\mathrm{R}$ diagram the primary lies almost exactly part way between the solar-metallicity ZAMS and TAMS lines, and the secondary slightly beyond the TAMS line, both being in comparable positions with the stars of the sample of Hilditch et al. (1988). An immediate conclusion strengthened from these results is that RU UMi is definitely a FO Virginistype of near-contact binary system rather than a V1010 Ophiuchi-type one, in accordance with the photometric property of having equally bright maxima. It even shares with FO Virginis itself an equally evolved secondary (in the $\mathrm{H}-\mathrm{R}$ diagram the secondaries also occupy almost the same position) and a very bright and massive primary, although less evolved in the case of RU UMi. If the generalization by Shaw (1990) holds true, it should moreover exhibit a lack of period change. 


\section{Discussion and conclusions}

From the results obtained using the Wilson-Devinney code it seems more probable that the system of RU UMi is semi-detached, with the secondary filling its inner Roche lobe, which in the case of synchronous rotation and circular orbit coincides with its limiting lobe. The primary must also be near the limits of its lobe, because the code tended to give values for the $\Omega_{1}$ potential close to the $\Omega_{\text {(in) }}$ value for the final mass ratio we derived. The system is a FO Virginis-type of near-contact binary. Concerning the uniqueness of our result, solutions with different spot combinations may equally well fit the observed light curves. This is a general problem of light curve fitting with spot models. Because of the non-uniqueness of such solutions, we refrained here from trying further spot combinations. As far as the evolutionary state of the system is concerned, the similarity with the A-type W UMa contact binaries, as supported also by this study, suggests that the FO Vir near-contact systems are either precursors or temporarily detached stages of the A-type W UMa systems.

Acknowledgements. We thank the referee Dr. R.W. Hilditch for his valuable comments on an earlier version of the manuscript. We also express our thanks to the staff of the Kryonerion Observatory for their help during the observations. The 3-D pictures were made with Binary Maker 2.0.

\section{References}

Bell, S. A., Hilditch, R. W., \& Edwin, R. P. 1993, MNRAS, 260, 478

Bradstreet, D. H. 1993, Binary Maker 2.0 User Manual

De Bernardi, C., \& Scaltriti, F. 1977, AcA, 27, 187

Hilditch, R. W., King, D. J., \& McFarlane, T. M. 1988, MNRAS, 231, 341

Hill, G., Hilditch, R. W., Younger, F., \& Fisher, W. A. 1975, Mem. Roy. Astron. Soc., 79, 131

Kaluzny, J. 1985, AcA, 35, 327

Kholopov, P. N., Samus', N. N, Frolov, M. S., et al. 1987, General Catalogue of Variable Stars, IV ed., v. III, "Nauka", 1987

Kwee, K. K., \& van Woerden, H. 1956, Bull. Astron. Inst. Netherlands, 12, 327

Maxted, P. F. L., \& Hilditch, R. W. 1996, The Observatory, 116,288

Nha, I.-S. 1973, AJ, 78, 107

Nakamura, Y., Yamasaki, A., \& Okazaki, A. 1988, PASJ, 40, 79

Rafert, J. B. 1990, AJ, 100, 1253

Shaw, J. S. 1990, in Active Close Binaries, ed. C. Ibanoglu (Kluwer Academic Publishers, Dordrecht), 241

Shaw, J. S. 1994, Mem. S. A. Ital., 65-1, 95

Strohmeier, W., \& Knigge, R. 1960, Bamberg Ver. Remeis, 5

Van Hamme, W. 1993, AJ, 106, 2096

Wood, D. B. 1971, PASP, 83, 286 